Association de loi 1901 Fondée en 1994

Unité - Solidarité - Convivialité Non à la discrimination Information sur l'ODF

Omnipratique et ODF : prévention - traitement Développement de l'ODF Formation Continue en ODF

Secrétariat :
37 rue d'Amsterdam
75008 PARIS
Tel : 0607038810
uniodf@uniodf.org
Fax : 0170790571
http ://www.uniodf.org
Retrouvez toutes les informations et
l'actualité orthodontiques en direct
sur www.uniodf.org.
Partagez avec nous vos expériences
sur notre forum :
Écrire un message :
uniodf@googlegroups.com
S'inscrire :
http :/groups.google.fr/group/uniodf?lnk=srg

Directeur de publication

Antoine HAROUN

Conception graphique et réalisation : Jérôme TERRIEN

\title{
NOUS SOMMES TOUS EUROPÉENS
}

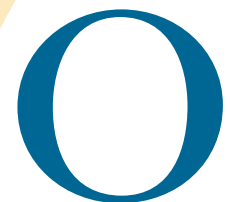

n l'a vu pour la chirurgie buccale, la France était un des derniers pays européens à ne pas avoir cette spécialité, les universitaires et l'Ordre National en rêvaient, les syndicats viennent de se rallier à cette idée en traînant les pieds et finalement la voilà sur les rails poussée par le vent communautaire. D'aucuns se réjouiront, d'autres s'inquièteront d'un possible appauvrissement de notre formation initiale et du champ de nos compétences de généralistes. Lisez sur ce point l'interview du Pr Marc BOLLA.

Mais les lois européennes peuvent aussi servir nos convictions et aller parfois à l'encontre de notre code de déontologie.

Ainsi l'article 10 de la Convention européenne de sauvegarde des droits de l'homme et des libertés fondamentales peut être invoqué dès lors que nos lois portent une atteinte excessive à la liberté de recevoir ou de communiquer des informations.

Par 2 arrêts rendus le 16 avril 2008, le Conseil d'Etat a considéré que l'interdiction faite à un chirurgien-dentiste de mentionner sur sa plaque d'autres titres ou diplômes que son diplôme d'état et sa spécialité et notamment ses compétences était contraire aux stipulations de l'article 10. Le premier Ministre a 6 mois pour édicter des mesures qui doivent mettre fin à cette situation...

Il devrait en être de même pour les exercices exclusifs.

Concernant leur mention, il faut rappeler que les dispositions du décret de 22 juillet 1967 interdisaient déjà aux chirurgiens-dentistes d'inscrire sur leurs imprimés professionnels, dans les annuaires et sur leur plaque professionnelle d'autres mentions que « leur spécialité reconnue dans les conditions déterminées par le conseil national de l'ordre»

Le Conseil National de l'Ordre ne s'est pas opposé avant 1994 à ce que les chirurgiens-dentistes qui en avaient fait la déclaration à leur conseil départemental et qui s'étaient engagés exclusivement à une discipline, le mentionnent sur les documents et plaques professionnels mais un arrêt du Conseil d'Etat du 26 novembre 1997 a mis fin aux tolérances consenties. Cet arrêt sur lequel s'appuient dorénavant tous les conseils départementaux pour ne pas permettre aux exclusifs odf d'informer leur patientèle de leur exercice particulier se trouve donc également en contradiction avec l'article 10 sus cité.

Notre exercice évoluera avec l'Europe, nous revendiquons un droit à l'information de nos patients. Omnipraticiens ou exclusifs, il serait légitime de pouvoir informer nos patients de nos compétences et de nos diplômes universitaires en ODF mais n'oublions pas que la qualité de nos soins et le bouche à oreille sont nos principaux atouts.

\section{Graphy Prim' imprimeur}

44300 NANTES 